\title{
On the integrability of a tritrophic food chain model
}

\author{
Jaume* Llibre \\ Departament de Matemàtiques, Universitat Autònoma de Barcelona, \\ 08193 Bellaterra, Barcelona, Catalonia, Spain \\ e-mail: jllibre@mat.uab.cat \\ Clàudia $^{\dagger}$ Valls \\ Departamento de Matemática, Instituto Superior Técnico, \\ 1049-001 Lisboa, Portugal. \\ e-mail: cvalls@math.ist.utl.pt
}

September 3, 2009

\begin{abstract}
In this paper we work with a vastly analyzed tritrophic food chain model. We provide a complete characterization of their Darboux polynomials and of their exponential factors. We also show the non-existence of polynomial first integrals, of rational first integrals, of local analytic first integrals in a neighborhood of the origin, of first integrals that can be described by formal series and of Darboux first integrals.
\end{abstract}

1991 Mathematics Subject Classification. 34C05, 34A34, 34C14.

Key words. Formal integrability, Darboux integrability, polynomial integrability, analytic integrability, tritrophic food chain model, Darboux polynomial, exponential factor

\section{Introduction}

During the last 80 years and after the works of Lotka [17] and Volterra [23] one of the main topics in mathematical ecology has been the study of ditrophic food chains by analyzing a big number of second-order differential equations (see for instance

*The author has been supported by the grants MEC/FEDER MTM 2008-03437 and CIRIT 2009SGR 410 .

${ }^{\dagger}$ The author has been partially supported by FCT through CAMGSD, Lisbon. 
[2]). Recently some interest appears in the search of tritrophic food chain models composed by prey, predator and top-predator. We refer the reader to $[11,12,10]$ and the references therein. The model that we consider in this paper describes a tritrophic food chain composed of a logistic prey $x$, a Holling type II predator $y$ and a Holling type II top-predator $z$. After rescaling of the variables it is given by the following system of ordinary differential equations (see $[13,20,14,19]$ for more details):

$$
\begin{aligned}
x^{\prime} & =x\left(\rho-\frac{x}{k}-\frac{a_{1} y}{b_{1}+x}\right), \\
y^{\prime} & =y\left(\frac{a_{1} x}{b_{1}+x}-\frac{a_{2} z}{b_{2}+y}-d_{1}\right), \\
z^{\prime} & =z\left(\frac{a_{2} y}{b_{2}+y}-d_{2}\right) .
\end{aligned}
$$

In order to preserve the biological meaning of the model, the 8 parameters of this system are assumed to be strictly positive. After a rescaling of time by $\left(b_{1}+x\right)\left(b_{2}+y\right)$ system (1.1) becomes the polynomial differential system

$$
\begin{aligned}
x^{\prime} & =x\left(\rho\left(b_{1}+x\right)\left(b_{2}+y\right)-\frac{x}{k}\left(b_{1}+x\right)\left(b_{2}+y\right)-a_{1} y\left(b_{2}+y\right)\right), \\
y^{\prime} & =y\left(a_{1} x\left(b_{2}+y\right)-a_{2} z\left(b_{1}+x\right)-d_{1}\left(b_{1}+x\right)\left(b_{2}+y\right)\right), \\
z^{\prime} & =z\left(a_{2} y\left(b_{1}+x\right)-d_{2}\left(b_{1}+x\right)\left(b_{2}+y\right)\right) .
\end{aligned}
$$

One of the main tools for studying the dynamics of a differential system like system (1.2) is to know the existence of first integrals for some values of the parameters. The existence of one first integral reduces the complexity of the dynamics of the system and the existence of two first integrals solve completely the problem. In general for a given differential system it is a difficult problem to determine the existence or non-existence of first integrals.

The aim of this paper is to study the existence of first integrals of system (1.2) that can be described by formal series or by rational functions by using the Darboux theory of integrability (see [6], [4] and [8]). The use of formal series in the study of differential equations and, in particular for studying the existence of analytic first integrals is a classical tool. Then, for instance, solutions described by formal series around singularities have been studied by Siedenberg [22], the existence of first integrals given by formal series have been studied by Nemytskii and Stepanov [21], Moussu [18], .... However the greatest success in using formal series to study differential equations has been achieved by Écalle [9] who used them to prove the Dulac's conjecture.

A polynomial first integral $f=f(x, y, z)$ of system (1.2) is a polynomial in the 
variables $x, y$ and $z$ such that

$$
\begin{aligned}
& x\left[\rho\left(b_{1}+x\right)\left(b_{2}+y\right)-\frac{x}{k}\left(b_{1}+x\right)\left(b_{2}+y\right)-a_{1} y\left(b_{2}+y\right)\right] \frac{\partial f}{\partial x}+ \\
& y\left[a_{1} x\left(b_{2}+y\right)-a_{2} z\left(b_{1}+x\right)-d_{1}\left(b_{1}+x\right)\left(b_{2}+y\right)\right] \frac{\partial f}{\partial y}+ \\
& z\left(a_{2} y\left(b_{1}+x\right)-d_{2}\left(b_{1}+x\right)\left(b_{2}+y\right)\right) \frac{\partial f}{\partial z}=0 .
\end{aligned}
$$

A formal first integral $f=f(x, y, z)$ of system (1.2) is a formal power series in the variables $x, y$ and $z$ such that $f$ satisfies (1.3).

A global analytic first integral $f=f(x, y, z)$ of system (1.2) is a function $f: \mathbb{R}^{3} \rightarrow$ $\mathbb{R}$ which is analytic in the variables $x, y$ and $z$ such that $f$ satisfies (1.3).

A local analytic first integral $f=f(x, y, z)$ of system (1.2) is an analytic function $f$ defined only in a neighborhood of some point of $\mathbb{R}^{3}$ satisfying (1.3).

A rational first integral of system (1.2) is a rational function $f$ satisfying (1.3).

The first main results of this paper are the following ones.

Theorem 1.1. System (1.2) has no formal first integrals.

An immediate consequence of Theorem 1.1 is the next result.

Corollary 1.1. System (1.2) has neither global analytic first integrals, nor polynomial first integrals, nor local analytic first integrals at the origin.

Theorem 1.2. System (1.2) has no rational first integrals.

To prove Theorem 1.2 we will use the Darboux theory of integrability. The Darboux theory of integrability in dimension 3 is based on the existence of invariant algebraic surfaces (or Darboux polynomials) and on the existence of exponential factors (that control the multiplicity of the invariant algebraic surfaces). For more details see [3]-[7] and [16]. This theory is one of the best theories for studying the existence of first integral for the polynomial differential systems.

A Darboux polynomial of system (1.2) is a polynomial $f \in \mathbb{C}[x, y, z] \backslash \mathbb{C}$ such that

$$
\begin{aligned}
& x\left(\rho\left(b_{1}+x\right)\left(b_{2}+y\right)-\frac{x}{k}\left(b_{1}+x\right)\left(b_{2}+y\right)-a_{1} y\left(b_{2}+y\right)\right) \frac{\partial f}{\partial x}+ \\
& y\left(a_{1} x\left(b_{2}+y\right)-a_{2} z\left(b_{1}+x\right)-d_{1}\left(b_{1}+x\right)\left(b_{2}+y\right)\right) \frac{\partial f}{\partial y}+ \\
& z\left(a_{2} y\left(b_{1}+x\right)-d_{2}\left(b_{1}+x\right)\left(b_{2}+y\right)\right) \frac{\partial f}{\partial z}=K f,
\end{aligned}
$$

for some polynomial

$$
\begin{aligned}
& K=\alpha_{0}+\alpha_{1} x+\alpha_{2} y+\alpha_{3} z+\alpha_{4} x^{2}+\alpha_{5} x y+\alpha_{6} x z+\alpha_{7} y^{2}+\alpha_{8} y z+\alpha_{9} z^{2}+ \\
& \alpha_{10} x^{3}+\alpha_{11} x^{2} y+\alpha_{12} x^{2} z+\alpha_{13} x y^{2}+\alpha_{14} x y z+\alpha_{15} x z^{2}+\alpha_{16} y^{3}+ \\
& \alpha_{17} y^{2} z+\alpha_{18} y z^{2}+\alpha_{19} z^{3} .
\end{aligned}
$$

Note that $f=0$ is an invariant algebraic hypersurface for the flow of system (1.2) and a polynomial first integral is a Darboux polynomial with zero cofactor. 
Theorem 1.3. The unique irreducible Darboux polynomials with non-zero cofactor are $x, y$ and $z$.

An exponential factor $F=F(x, y, z)$ of system (1.2) is a function $F=\exp (h / f) \notin$ $\mathbb{C}$ with $h, f \in \mathbb{C}[x, y, z]$ satisfying that

$$
\frac{\partial F}{\partial x} \dot{x}+\frac{\partial F}{\partial y} \dot{y}+\frac{\partial F}{\partial z} \dot{z}=L F,
$$

for some polynomial $L \in \mathbb{C}[x, y, z]$ of degree at most 3 , called the cofactor of $F$. Of course in equation (1.6) $\dot{x}, \dot{y}$ and $\dot{z}$ are the ones given in (1.2).

A Darboux first integral $G$ of system (1.2) is a first integral of the form

$$
G=f_{1}^{\lambda_{1}} \cdots f_{p}^{\lambda_{p}} F_{1}^{\mu_{1}} \cdots F_{q}^{\mu_{q}},
$$

where $f_{1}, \ldots, f_{p}$ are Darboux polynomials, $F_{1}, \ldots, F_{q}$ are exponential factors and $\lambda_{j}, \mu_{k} \in \mathbb{C}$ for $j=1, \ldots, p, k=1, \ldots, q$.

Theorem 1.4. The unique exponential factors of system (1.2) are of the form $e^{h}$ with $h=h(y, z)$ a polynomial. Its cofactor is

$$
L_{h}=\frac{\partial h}{\partial x} \dot{x}+\frac{\partial h}{\partial y} \dot{y}+\frac{\partial h}{\partial z} \dot{z} .
$$

(a) If $d_{1} \neq a_{1}$, then $e^{y}$ and $e^{z}$ are exponential factors, and all the other exponential factors are $e^{a y+b z}$ with $(a, b) \in \mathbb{C} \backslash\{(0,0)\}$. Moreover $L_{a y+b z}=a L_{y}+b L_{z}$.

(b) If $d_{1}=a_{1}$, then $e^{y}$, $e^{z}$ and $e^{h}$ with

$$
h=2 b_{2}\left(a_{2}-d_{2}\right)^{2} y-\left(a_{2}-d_{2}\right)^{2} y^{2}-2 a_{2}\left(a_{2}-d_{2}\right) y z-a_{2}^{2} z^{2},
$$

are exponential factors, and all the other exponential factors are $e^{a y+b z+c h}$ with $(a, b, c) \in \mathbb{C} \backslash\{(0,0,0)\}$. Moreover $L_{a y+b z+c h}=a L_{y}+b L_{z}+c L_{h}$. Note that if additionally $d_{2}=a_{2}$, then $h$ reduces to $z^{2}$.

Theorem 1.5. System (1.2) has no Darboux first integrals.

In Section 2 we state and prove preliminary results that will be used in the proofs of Theorems 1.4 and 1.5. In Section 3 we prove Theorems 1.4 and 1.5.

The paper is organized as follows. In Section 2 we prove Theorem 1.1 and Corollary 1.1. In Section 3 we prove Theorem 1.3 and in Section 4 we prove Theorem 1.2. Furthermore, in Section 5 we state and prove preliminary results that will be used in the proofs of Theorems 1.4 and 1.5. Finally, in Section 7 we prove Theorems 1.4 and 1.5 .

\section{Proof of Theorem 1.1 and Corollary 1.1}

Proof of Theorem 1.1. Let $f$ be a formal first integral of system (1.2). Without loss of generality we can assume that $f$ has no constant terms. Then $f$ satisfies (1.3). 
We write $f=\sum_{j \geq 0} f_{j}(x, z) y^{j}$, where every $f_{j}(x, z)$ is a formal power series in the variables $x$ and $z$. We consider two cases:

Case 1: $f$ is not divisible by $y$. In this case we have that $f_{0}=f_{0}(x, z) \neq 0$ is a formal first integral of system (1.2) restricted to $y=0$. System (1.2) restricted to $y=0$ becomes

$$
x^{\prime}=\left(b_{1}+x\right) b_{2} x\left(\rho-\frac{x}{k}\right), \quad z^{\prime}=-\left(b_{1}+x\right) b_{2} d_{2} z .
$$

Then, if $f_{0}$ is a formal first integral of (2.1) after rescaling the time by $b_{2}\left(b_{1}+x\right)$ we obtain that $f_{0}$ is a first integral of the system

$$
x^{\prime}=x\left(\rho-\frac{x}{k}\right), \quad z^{\prime}=-d_{2} z .
$$

It is easy to check that all the first integrals of system (2.2) are functions in the variable $G=(x-k) z^{-\rho / d_{2}} / x$. Since $k, \rho$ and $d_{2}$ are positive, any function in the variable $G$ cannot produce a formal power series in the variables $x$ and $z$. So $f_{0}$ is not a formal first integral of system (2.2), and we have a contradiction.

Case 2: $f$ is divisible by $y$. Then $f=y^{l} g$ with $l \geq 1$ and $g$ is not divisible by $y$. Then, from (1.3), after simplifying by $y^{l}$ we get that $g$ satisfies

$$
\begin{aligned}
& x\left[\rho\left(b_{1}+x\right)\left(b_{2}+y\right)-\frac{x}{k}\left(b_{1}+x\right)\left(b_{2}+y\right)-a_{1} y\left(b_{2}+y\right)\right] \frac{\partial g}{\partial x}+ \\
& y\left[a_{1} x\left(b_{2}+y\right)-a_{2} z\left(b_{1}+x\right)-d_{1}\left(b_{1}+x\right)\left(b_{2}+y\right)\right] \frac{\partial g}{\partial y}+ \\
& z\left(a_{2} y\left(b_{1}+x\right)-d_{2}\left(b_{1}+x\right)\left(b_{2}+y\right)\right) \frac{\partial g}{\partial z}= \\
& -l\left(a_{1} x\left(b_{2}+y\right)-a_{2} z\left(b_{1}+x\right)-d_{1}\left(b_{1}+x\right)\left(b_{2}+y\right)\right) g
\end{aligned}
$$

Now we write $g=\sum_{j \geq 0} g_{j}(x, z) y^{j}$. We have that every $g_{j}=g_{j}(x, z)$ is a formal power series in the variables $x$ and $z$. Furthermore $g_{0} \neq 0$, otherwise $g$ would be divisible by $y$, a contradiction. Moreover $g_{0}$ satisfies (2.3) restricted to $y=0$ that is,

$$
b_{2}\left(b_{1}+x\right)\left[x\left(\rho-\frac{x}{k}\right) \frac{\partial g_{0}}{\partial x}-d_{2} z \frac{\partial g_{0}}{\partial z}\right]=-l\left(a_{1} x b_{2}-a_{2} z\left(b_{1}+x\right)-d_{1} b_{2}\left(b_{1}+x\right)\right) g_{0} .
$$

Now we consider two cases.

Case 2.1: $g_{0}$ is not divisible by $b_{1}+x$. In this case since $g_{0} \neq 0$ and $a_{1} b_{2}>0$, from (2.4) we get a contradiction.

Case 2.2: $g_{0}$ is divisible by $b_{1}+x$. We write $g_{0}=\left(b_{1}+x\right)^{m} h$ with $m \geq 1$ and $h=h(x, z)$ being a formal power series in the variables $x, z$ that is not divisible by $b_{1}+x$. Then from (2.4) after simplifying by $\left(b_{1}+x\right)^{m}$ we obtain that $h$ must satisfy

$$
\begin{aligned}
& b_{2}\left(b_{1}+x\right)\left[x\left(\rho-\frac{x}{k}\right) \frac{\partial h}{\partial x}-d_{2} z \frac{\partial h}{\partial z}\right]= \\
& -\left[b_{2}\left(l a_{1}+m \rho\right) x-a_{2} l z\left(b_{1}+x\right)-b_{2} l\left(b_{1}+x\right) d_{1}-m b_{2} \frac{x^{2}}{k}\right] h .
\end{aligned}
$$


Since $b_{2} m / k>0$ and $b_{2}\left(l a_{1}+m \rho\right)>0$ we have from (2.5) that $h$ must be divisible by $b_{1}+x$ a contradiction. This is the end of the proof.

Proof of Corollary 1.1. We proceed by contradiction. Assume that $g$ is an analytic first integral of system (1.2) in a neighborhood $U$ of the origin. Clearly there exists a neighborhood $V$ of the system contained in $U$ such that $g_{\mid V}$ can be written as a formal power series which converges. Hence in $V g$ is a formal first integral of system (1.2), a contradiction with Theorem 1.1.

Clearly if system (1.2) has a global analytic first integral or a polynomial first integral it has a local analytic first integral in a neighborhood of the origin. So the proof of Corollary 1.1 in these cases is reduced to the above proof. This is the end of the proof.

\section{Darboux polynomials with non-zero cofactor}

The proof of Theorem 1.3 is divided into several lemmas. We denote by $\mathbb{Z}^{+}$the set of non-negative integers.

Lemma 3.1. Let $f=f(x, y, z)$ be a homogeneous polynomial of degree $n$ satisfying

$$
P(x, y) \frac{\partial f}{\partial x}=Q(x, y, z) f
$$

where $P(x, y)$ is a polynomial of degree $k \geq 1$ in the variables $x, y$ and $Q(x, y, z)$ is a polynomial in the variables $x, y, z$ of degree $k-1$ which is not divisible by $P(x, y)$ and such that for any $m \in \mathbb{Z}^{+}, Q(x, y, z)-m \frac{\partial P(x, y)}{\partial x}$ is not divisible by $P(x, y)$. Then $f=0$.

Proof. We proceed by contradiction. We assume that $f \neq 0$ and we will reach a contradiction. We consider two cases. Since $P$ does not divide $Q, P$ divides $f$. Therefore we write $f=P^{m} g$, where $m \geq 1$ and $\operatorname{deg}(g)=n-m \operatorname{deg} P$ and $g$ is not divisible by $P$. It follows from (3.1) after simplifying by $P^{m}$ that $g$ satisfies

$$
-\frac{1}{k} P(x, y) \frac{\partial g}{\partial x}=\left(Q(x, y, z)-m \frac{\partial P}{\partial x}\right) g
$$

Then from (3.2) taking into account that $P$ does not divide $Q-m \frac{\partial P}{\partial x}$ we get that $P$ divides $g$, a contradiction. This is the end of the proof.

Lemma 3.2. Let $f$ be an irreducible Darboux polynomial of degree $\geq 2$ with a non-zero cofactor $K$ as in (1.5). Then

$$
\alpha_{10}=\alpha_{12}=\alpha_{13}=\alpha_{14}=\alpha_{15}=\alpha_{16}=\alpha_{17}=\alpha_{18}=\alpha_{19}=0 .
$$

Proof. Suppose that $f$ has degree $n \geq 2$. We write $f=\sum_{j=0}^{n} f_{j}$ where each $f_{j}=$ $f_{j}(x, y, z)$ is a homogeneous polynomial of degree $j$. Clearly $f_{n} \neq 0$. Computing 
the terms of degree $n+3$ in (1.4) we have that

$$
-\frac{1}{k} x^{3} y \frac{\partial f_{n}}{\partial x}=K_{3} f_{n}
$$

where

$$
\begin{aligned}
& K_{3}=\alpha_{10} x^{3}+\alpha_{11} x^{2} y+\alpha_{12} x^{2} z+\alpha_{13} x y^{2}+\alpha_{14} x y z+\alpha_{15} x z^{2}+\alpha_{16} y^{3}+\alpha_{17} y^{2} z+ \\
& \alpha_{18} y z^{2}+\alpha_{19} z^{3} .
\end{aligned}
$$

Note that $\operatorname{deg}\left(K_{3}\right)=3$. We separate the proof in two cases.

Case 1: $K_{3}$ is not divisible by $x$. If in addition $K_{3}$ is not divisible by $y$, then from (3.3) using Lemma 3.1 with $P(x, y)=x^{3} y$ and $Q(x, y)=K_{3}$ we get that $f_{n}=0$ a contradiction. Thus $K_{3}$ is divisible by $y$. We write $K_{3}=y \hat{K}_{3}$. Note that $\hat{K}_{3}$ has not $x$ as a factor. We obtain that (3.3) becomes

$$
-\frac{1}{k} x^{3} \frac{\partial f_{n}}{\partial x}=\hat{K}_{3} f_{n}
$$

In this case from (3.5) using Lemma 3.1 with $P(x, y)=x^{3}$ and $Q(x, y)=\hat{K}_{3}$ we get that $f_{n}=0$ a contradiction.

Case 2: $K_{3}$ is divisible by $x$. We write $K_{3}=x \hat{K}_{3}$ with $\operatorname{deg}\left(\hat{K}_{3}\right)=2$. Then (3.3) becomes

We consider two subcases.

$$
-\frac{1}{k} x^{2} y \frac{\partial f_{n}}{\partial x}=\hat{K}_{3} f_{n}
$$

Subcase 2.1: $\hat{K}_{3}$ is not divisible by $x$. Then repeating the arguments of Case 1 we arrive to a contradiction.

Subcase 2.2: $\hat{K}_{3}$ is divisible by $x$. We write $\hat{K}_{3}=x \tilde{K}_{3}$ with $\operatorname{deg}\left(\tilde{K}_{3}\right)=1$. Then (3.6) becomes

$$
-\frac{1}{k} x y \frac{\partial f_{n}}{\partial x}=\tilde{K}_{3} f_{n}
$$

We first assume that $\tilde{K}_{3}$ is divisible by $x$. Then $\tilde{K}_{3}=x \bar{K}_{3}$ with $\bar{K}_{3} \in \mathbb{C}$. Then

$$
-\frac{1}{k} y \frac{\partial f_{n}}{\partial x}=\bar{K}_{3} f_{n}
$$

and thus $f_{n}$ must be divisible by $y$. Therefore we can write $f_{n}=y^{l} g$, with $l \geq 1$, $\operatorname{deg}(g)=n-l, g$ is not divisible by $y$ and satisfies, after simplifying by $y^{l}$, equation (3.8) with $f_{n}$ replaced by $g$. Thus $g$ must be divisible by $y$ a contradiction.

Then $\tilde{K}_{3}$ is not divisible by $x$. Furthermore, if $\tilde{K}_{3}$ is not divisible by $y$, by (3.7) and using Lemma 3.1 with $P(x, y)=x y$ and $Q(x, y)=\tilde{K}_{3}$ we get that $f_{n}=0$ a contradiction. Thus $\tilde{K}_{3}$ must be divisible by $y$, that is, $\tilde{K}_{3}=y \bar{K}_{3}$ with $\bar{K}_{3} \in \mathbb{C}$. So $K_{3}=\bar{K}_{3} x^{2} y$. By (3.4) we have that $\alpha_{10}=\alpha_{12}=\alpha_{13}=\alpha_{14}=\alpha_{15}=\alpha_{16}=\alpha_{17}=$ $\alpha_{18}=\alpha_{19}=0$. This is the end of the proof.

In view of Lemma 3.2 we get that

$$
K=\alpha_{0}+\alpha_{1} x+\alpha_{2} y+\alpha_{3} z+\alpha_{4} x^{2}+\alpha_{5} x y+\alpha_{6} x z+\alpha_{7} y^{2}+\alpha_{8} y z+\alpha_{9} z^{2}+\alpha_{11} x^{2} y .
$$


Lemma 3.3. Let $f$ be an irreducible Darboux polynomial of degree $\geq 2$ with a non-zero cofactor $K$ as in (3.9). Then

$$
\alpha_{0}=\alpha_{3}=\alpha_{6}=\alpha_{9}=0, \quad \alpha_{1}=\frac{b_{2}}{k}\left(n_{1} k \rho-n_{2} b_{1}\right), \quad \alpha_{4}=-\frac{b_{2}}{k}\left(n_{1}+n_{2}\right),
$$

for some $n_{1}, n_{2} \in \mathbb{Z}^{+}$.

Proof. Assume that $f$ has degree $n \geq 2$. We write $f=\sum_{j=1}^{n} f_{j} y^{j}$ where each $f_{j}=$ $f_{j}(x, z)$ is a polynomial in the variables $x$ and $z$. Since $f$ is irreducible, we get that $f_{0} \neq 0$. Furthermore $f_{0}$ is a Darboux polynomial of system (1.2) restricted to $y=0$ with cofactor $K$ as in (3.9) restricted to $y=0$, i.e.,

$$
\bar{K}=\alpha_{0}+\alpha_{1} x+\alpha_{3} z+\alpha_{4} x^{2}+\alpha_{6} x z+\alpha_{9} z^{2} .
$$

Then by (1.4)

$$
b_{2}\left(b_{1}+x\right)\left[x\left(\rho-\frac{x}{k}\right) \frac{\partial f_{0}}{\partial x}-d_{2} z \frac{\partial f_{0}}{\partial z}\right]=\bar{K} f_{0} .
$$

Let $m=\operatorname{deg}\left(f_{0}\right)$. We assume that $\alpha_{9} \neq 0$ or $\alpha_{3} \neq b_{1} \alpha_{6}$. This implies that $b_{1}+x$ cannot divide $\bar{K}$. Therefore, by (3.10) we obtain that $f_{0}$ must be divisible by $b_{1}+x$. We have then that $f_{0}=\left(b_{1}+x\right)^{l} h$ with $1 \leq l \leq m, \operatorname{deg}(h)=m-l$ and $h$ is not divisible by $b_{1}+x$. From (3.10) and after simplifying by $\left(b_{1}+x\right)^{l}$ we obtain

$$
b_{2}\left(b_{1}+x\right)\left[x\left(\rho-\frac{x}{k}\right) \frac{\partial h}{\partial x}-d_{2} z \frac{\partial h}{\partial z}\right]=\left(\bar{K}-l b_{2} x\left(\rho-\frac{x}{k}\right)\right) h .
$$

By hypothesis we have that $b_{1}+x$ does not divide $\bar{K}-l b_{2} x\left(\rho-\frac{x}{k}\right)$. Then by (3.11) $h$ must be divisible by $b_{1}+x$, a contradiction. Hence,

$$
\bar{K}=\alpha_{0}+\alpha_{1} x+\alpha_{6} z\left(b_{1}+x\right)+\alpha_{4} x^{2} .
$$

Now if we restrict (3.10) to $x=0$ and denote by $g$ the restriction of $f_{0}$ to $x=0$ we obtain

$$
-b_{1} b_{2} d_{2} z \frac{d g}{d z}=\left(\alpha_{0}+\alpha_{6} b_{1} z\right) g
$$

Hence

$$
g=L e^{-\frac{\alpha_{6} z}{b_{2} d_{2}}} z^{-\frac{\alpha_{0}}{b_{1} b_{2} d_{2}}} \quad \text { with } \quad L \in \mathbb{C} .
$$

Since $g$ must be a polynomial and $b_{1}, b_{2}, d_{2}>0$ we must have $\alpha_{6}=0$ and $\alpha_{0}=$ $-j b_{1} b_{2} d_{2}$ with $j \in \mathbb{Z}^{+}$, and thus $\alpha_{3}=0$.

From (3.12) we have $\bar{K}=-j b_{1} b_{2} d_{2}+\alpha_{1} x+\alpha_{4} x^{2}$. Restricting equation (3.10) to $z=0$ and denoting by $h$ the restriction of $f_{0}$ to $z=0$ we obtain

$$
b_{2}\left(b_{1}+x\right) x\left(\rho-\frac{x}{k}\right) \frac{d h}{d x}=\left(-j b_{1} b_{2} d_{2}+\alpha_{1} x+\alpha_{4} x^{2}\right) h,
$$


and thus

$$
h=L x^{-\frac{j d_{2}}{\rho}}\left(b_{1}+x\right)^{\frac{\left.k\left(\alpha_{1}-\alpha_{4} b_{1}\right)+b_{2} d_{2} j\right)}{b_{2}\left(b_{1}+k \rho\right)}}(x-\rho k)^{\frac{j b_{1} b_{2} d_{2}-k \rho\left(\alpha_{1}+\alpha_{4} k \rho\right)}{b_{2} \rho\left(b_{1}+k \rho\right)}}, \quad L \in \mathbb{C} .
$$

Since $d_{2}, \rho>0$ and $h$ must be a polynomial we get that $j=0$ (i.e. $\alpha_{0}=0$ ) and that

$$
\alpha_{1}=\frac{b_{2}}{k}\left(n_{1} k \rho-n_{2} b_{1}\right), \quad \alpha_{4}=-\frac{b_{2}}{k}\left(n_{1}+n_{2}\right),
$$

for some $n_{1}, n_{2} \in \mathbb{Z}^{+}$. This is the end of the proof.

In view of Lemma 3.3 we get that

$$
K=\frac{b_{2}}{k}\left(n_{1} k \rho-n_{2} b_{1}\right) x-\frac{b_{2}}{k}\left(n_{1}+n_{2}\right) x^{2}+y\left(\alpha_{2}+\alpha_{5} x+\alpha_{7} y+\alpha_{8} z+\alpha_{11} x^{2}\right) .
$$

Lemma 3.4. Let $f$ be an irreducible Darboux polynomial of degree $\geq 2$ with nonzero cofactor $K$ as in (3.13). Then

$$
\alpha_{7}=\alpha_{8}=0, \quad \alpha_{2}=-n_{3} b_{1} d_{1},
$$

for some $n_{3} \in \mathbb{Z}^{+}$.

Proof. Assume that $f$ has degree $n \geq 2$. We write $f=\sum_{j=1}^{n} f_{j} x^{j}$ where each $f_{j}=$ $f_{j}(y, z)$ is a polynomial in the variables $y$ and $z$. Then $f_{0} \neq 0$, otherwise $f$ would be divisible by $x$, a contradiction. Furthermore $f_{0}$ is a Darboux polynomial of system (1.4) restricted to $x=0$, that is

$$
b_{1} y\left(-a_{2} z-d_{1}\left(b_{2}+y\right)\right) \frac{\partial f_{0}}{\partial y}+b_{1} z\left(a_{2} y-d_{2}\left(b_{2}+y\right)\right) \frac{\partial f_{0}}{\partial z}=y\left(\alpha_{2}+\alpha_{7} y+\alpha_{8} z\right) f_{0} .
$$

Assume $\alpha_{7}, \alpha_{8} \neq 0$. We write $f_{0}=\sum_{j=1}^{m} f_{0, j}$ where each $f_{0, j}$ is a homogeneous polynomial in the variables $y$ and $z$ of degree $j$ and $0 \leq m \leq n$. Clearly $f_{0, m} \neq 0$ because $f_{0} \neq 0$. Computing the terms of degree $m+2$ in (3.14) we get

$$
y\left(\alpha_{7} y+\alpha_{8} z\right) f_{0, m}=0,
$$

and since $\alpha_{7}, \alpha_{8} \neq 0$, we deduce $f_{0, m}=0$, a contradiction. Hence $\alpha_{7}=\alpha_{8}=0$.

Now we restrict (3.14) to $z=0$ and we denote by $h$ the restriction of $f_{0}$ to $z=0$. Then after simplifying by $y$ we have

$$
-\left(b_{2}+y\right) d_{1} b_{1} \frac{d h}{d y}=\alpha_{2} h, \quad \text { that is } \quad g=L\left(b_{2}+y\right)^{-\frac{\alpha_{2}}{d_{1} b_{1}}} \quad \text { with } \quad L \in \mathbb{C} .
$$

Then $\alpha_{2}=-n_{3} b_{1} d_{1}$ for some $n_{3} \in \mathbb{Z}^{+}$. This is the end of the proof.

In view of Lemma 3.4 we have that

$$
K=\frac{b_{2}}{k}\left(n_{1} k \rho-n_{2} b_{1}\right) x-\frac{b_{2}}{k}\left(n_{1}+n_{2}\right) x^{2}+y\left(-n_{3} b_{1} d_{1}+\alpha_{5} x+\alpha_{11} x^{2}\right) .
$$


Lemma 3.5. Let $f$ be an irreducible Darboux polynomial of degree $\geq 2$ with non zero cofactor $K$ as in (3.15). Then

$$
n_{1}=n_{2}=0, \quad \alpha_{11}=0 \quad \text { and } \quad \alpha_{5}=n_{3}\left(a_{1}-d_{1}\right),
$$

for $n_{3} \in \mathbb{Z}^{+}$as in (3.15).

Proof. Assume that $f$ has degree $n \geq 2$. We write $f=\sum_{j=1}^{n} f_{j} z^{j}$ where each $f_{j}=$ $f_{j}(x, y)$ is a polynomial in the variables $x$ and $y$. Since $f$ is irreducible we deduce that $f_{0} \neq 0$. Moreover $f_{0}$ is a Darboux polynomial of system (1.2) restricted to $z=0$, that is

$$
\left(b_{2}+y\right)\left(x\left(\rho\left(b_{1}+x\right)-\frac{x}{k}\left(b_{1}+x\right)-a_{1} y\right) \frac{\partial f_{0}}{\partial x}+y\left(a_{1} x-d_{1}\left(b_{1}+x\right)\right) \frac{\partial f_{0}}{\partial y}\right)=K f_{0},
$$

where $K$ has given in (3.15). Let $m=\operatorname{deg}\left(f_{0}\right)$. Assume that $\alpha_{11} \neq-\left(n_{1}+n_{2}\right) / k$ or $\alpha_{5} \neq\left(n_{1} k \rho-n_{2} b_{1}\right) / k+n_{3}\left(a_{1}-d_{1}\right)$. This implies that $b_{2}+y$ cannot divide $K$ because if $\alpha_{11} \neq-\left(n_{1}+n_{2}\right) / h$ it is clear, if $n_{3} \neq 0$ also it is clear, and if $n_{3}=0$ and $\alpha_{5} \neq\left(n_{1} k \rho-n_{2} b_{1}\right) / k$ again is clear. Therefore, by (3.16) we obtain that $f_{0}$ must be divisible by $b_{2}+y$. We have then that $f_{0}=\left(b_{2}+y\right)^{l} h$ with $1 \leq l \leq m$, $\operatorname{deg}(h)=m-l$ and $h$ is not divisible by $b_{2}+y$. After simplifying by $\left(b_{2}+y\right)^{l}$ we deduce that $h$ satisfies

$$
\begin{aligned}
& \left(b_{2}+y\right)\left(x\left(\rho\left(b_{1}+x\right)-\frac{x}{k}\left(b_{1}+x\right)-a_{1} y\right) \frac{\partial h}{\partial x}+y\left(a_{1} x-d_{1}\left(b_{1}+x\right)\right) \frac{\partial h}{\partial y}\right)= \\
& \left(K-l y\left(\left(a_{1}-d_{1}\right) x-d_{1} b_{1}\right)\right) h .
\end{aligned}
$$

By hypothesis we have that $b_{2}+y$ does not divide $\left.K-l y\left(\left(a_{1}-d_{1}\right) x-d_{1} b_{1}\right)\right)$ and by (3.17) $h$ must be divisible by $b_{2}+y$, a contradiction. Therefore we have

$$
\alpha_{11}=-\frac{1}{k}\left(n_{1}+n_{2}\right) \quad \text { and } \quad \alpha_{5}=\frac{1}{k}\left(n_{1} k \rho-n_{2} b_{1}\right)+n_{3}\left(a_{1}-d_{1}\right) .
$$

We assume that $n_{1}, n_{2} \neq 0$ and we will reach a contradiction. if $l<n_{3}$ and $n_{3} \neq 0$ then, from (3.17), $b_{2}+y$ divides $h$, a contradiction. So $l=n_{3}$ and $f_{0}=\left(b_{2}+y\right)^{n_{3}} h$ with $\operatorname{deg}(h)=m-n_{3}:=\bar{n}$. In view of (3.15), (3.17) with $l=n_{3}$ and (3.18) we get that $h$ satisfies, after simplifying by $b_{2}+y$, the equation

$$
\begin{aligned}
& x\left(\rho\left(b_{1}+x\right)-\frac{x}{k}\left(b_{1}+x\right)-a_{1} y\right) \frac{\partial h}{\partial x}+y\left(a_{1} x-d_{1}\left(b_{1}+x\right)\right) \frac{\partial h}{\partial y}= \\
& \frac{x}{k}\left(\left(n_{1} k \rho-n_{2} b_{1}\right)-\left(n_{1}+n_{2}\right) x\right) h .
\end{aligned}
$$

Now we write $h=\sum_{j=1}^{\bar{n}} h_{j}(x) y^{j}$ where each $h_{j}=h_{j}(x)$ is a polynomial of degree in the variable $x$. Computing the terms of degree $\bar{n}+1$ in the variable $y$ in (3.19) we obtain

$$
-a_{1} x \frac{d h_{\bar{n}}}{d x}=0 \quad \text { that is } \quad h_{\bar{n}}=c_{\bar{n}} \in \mathbb{C} .
$$


Now computing the terms of degree $\bar{n}$ in the variable $y$ in (3.19) we obtain

$$
-a_{1} x \frac{d h_{\bar{n}-1}}{d x}+\bar{n}\left(a_{1} x-d_{1}\left(b_{1}+x\right)\right) c_{\bar{n}}=\frac{x}{k}\left(\left(n_{1} k \rho-n_{2} b_{1}\right)-\left(n_{1}+n_{2}\right) x\right) c_{\bar{n}} .
$$

Evaluating (3.20) on $x=0$ we get that

$$
-\bar{n} d_{1} b_{1} c_{\bar{n}}=0 \quad \text { and thus } \quad c_{\bar{n}}=0, \quad \text { and } \quad h_{\bar{n}-1}=c_{\bar{n}-1} \in \mathbb{C} .
$$

Now proceeding for $h_{\bar{n}-1}$ as we did for $h_{\bar{n}}$ (changing $\bar{n}$ by $\bar{n}-1$ ) we get that $h_{\bar{n}-1}=c_{\bar{n}-1}=0$ and $h_{\bar{n}-2}=c_{\bar{n}-2} \in \mathbb{C}$. Therefore, doing this $\bar{n}$ times we get that $h=c_{0} \in \mathbb{C}$. By (3.19) and since $n_{1}, n_{2} \neq 0$, we get that $c_{0}=0$ and thus $f_{0}=0$, a contradiction. This implies that $n_{1}=n_{2}=0$. Then $\alpha_{11}=0$ and $\alpha_{5}=n_{3}\left(a_{1}-d_{1}\right)$. This concludes the proof of the lemma when $n_{3} \neq 0$.

Assume that $n_{3}=0$ and $n_{1}, n_{2} \neq 0$. Then, from (3.16) and (3.18) we obtain again (3.19) and the proof follows in a similar way. This is the end of the proof.

In view of Lemma 3.5 we have that

$$
K=n_{3} y\left(-b_{1} d_{1}+\left(a_{1}-d_{1}\right) x\right), \quad n_{3} \in \mathbb{Z}^{+} .
$$

Lemma 3.6. System (1.2) restricted to $y=0$ has no polynomial first integrals.

Proof. Note that system (1.2) restricted to $y=0$ and after the rescaling of the time by the quantity $b_{2}\left(b_{1}+x\right)$ can be written as

$$
x^{\prime}=x\left(\rho-\frac{x}{k}\right), \quad z^{\prime}=-d_{2} z .
$$

Then any first integral must be a function of $F=\frac{k \rho-x}{x} z^{-\rho / d_{2}}$ and any function of the variable $F$ cannot be a polynomial. This concludes the proof of the lemma. This is the end of the proof.

Lemma 3.7. Let $f$ be an irreducible Darboux polynomial of degree $\geq 2$ with nonzero cofactor $K$ as in (3.21). Then $n_{3}=0$.

Proof. We proceed by contradiction. We suppose $n_{3} \neq 0$. Assume that $f$ has degree $n \geq 2$. We write $f=\sum_{j=1}^{n} f_{j} y^{j}$ where each $f_{j}=f_{j}(x, z)$ is a polynomial in the variables $x$ and $z$. Since $f$ must be irreducible we deduce that $f_{0} \neq 0$. Moreover, $f_{0}$ is a Darboux polynomial of system (1.2) restricted to $y=0$, with cofactor $K$ as in (3.21) restricted to $y=0$. Since $K$ restricted to $y=0$ is equal to zero we have that

$$
b_{2}\left(b_{1}+x\right)\left[x\left(\rho-\frac{x}{k}\right) \frac{\partial f_{0}}{\partial x}-d_{2} z \frac{\partial f_{0}}{\partial z}\right]=0 .
$$

Clearly $f_{0}$ is either a constant or a polynomial first integral of system (1.2) restricted to $y=0$. In view of Lemma 3.6 this last case is not possible, hence it must be a constant. Thus

$$
f=c_{0}+y g \quad \text { where } c_{0} \in \mathbb{C} \text { and } g=g(x, y, z) \text { is a polynomial. }
$$


Note that $c_{0} \neq 0$ otherwise it would be irreducible, a contradiction. Then, after simplifying by $y, g$ satisfies

$$
\begin{aligned}
& x\left[\rho\left(b_{1}+x\right)\left(b_{2}+y\right)-\frac{x}{k}\left(b_{1}+x\right)\left(b_{2}+y\right)-a_{1} y\left(b_{2}+y\right)\right] \frac{\partial g}{\partial x}+ \\
& y\left[a_{1} x\left(b_{2}+y\right)-a_{2} z\left(b_{1}+x\right)-d_{1}\left(b_{1}+x\right)\left(b_{2}+y\right)\right] \frac{\partial g}{\partial y}+ \\
& z\left(a_{2} y\left(b_{1}+x\right)-d_{2}\left(b_{1}+x\right)\left(b_{2}+y\right)\right) \frac{\partial g}{\partial z}= \\
& n_{3}\left(-d_{1}\left(b_{1}+x\right)+a_{1} x\right)\left(c_{0}+y g\right)-\left(a_{1} x\left(b_{2}+y\right)-a_{2} z\left(b_{1}+x\right)-d_{1}\left(b_{1}+x\right)\left(b_{2}+y\right)\right) g .
\end{aligned}
$$

Now if we restrict equation (3.22) to $x=y=0$ and denote by $g_{0}$ the restriction of $g$ to $x=y=0$ we obtain, after simplifying by $b_{1}$, that

$$
-d_{2} b_{2} z \frac{d g_{0}}{d z}=-d_{1} n_{3} c_{0}+\left(a_{2} z+d_{1} b_{2}\right) g_{0} .
$$

Since $c_{0} n_{3} \neq 0$ we have that $g_{0} \neq 0$. Solving (3.23) we get that

$$
g_{0}=g_{0}(z)=e^{-a_{2} z /\left(b_{2} d_{2}\right)}\left(L z^{-d_{1} / d_{2}}-\frac{c_{0} d_{1} n_{3}}{b_{2} d_{2}} E_{\frac{d_{2}-d_{1}}{d_{2}}}\left(\frac{-a_{2} z}{b_{2} d_{2}}\right)\right),
$$

where $L \in \mathbb{C}$ and

$$
E_{l}(w)=\int_{1}^{\infty} \frac{e^{-w t}}{t^{l}} d t
$$

is the exponential integral function, see for more details [1]. Computing the limit of $g_{0}(z)$ given in (3.24) when $z \rightarrow \infty$ we get that

$$
\lim _{z \rightarrow \infty} g_{0}(z)=0
$$

a contradiction with the fact that $g_{0} \neq 0$ is a polynomial in the variable $z$. This is the end of the proof.

In view of Lemma 3.7 we have that $K=0$. In other words, there does not exist any irreducible Darboux polynomial of degree $\geq 2$ with non-zero cofactor.

Proof of Theorem 1.3. Clearly $x, y, z$ are irreducible Darboux polynomials of system (1.2) with non-zero cofactor. A direct easy but tedious computation using equation (1.4) allows to prove that if there are invariant planes $c_{0}+c_{1} x+c_{2} y+c_{3} z=0$ different from $x=0, y=0$ and $z=0$, then some of the eight parameters of system (1.2) are zero. Hence such invariant planes do not exist when the parameters are positive.

Moreover, in view of Lemmas 3.2-3.7 there are no Darboux polynomials of degree $\geq 2$ with non-zero cofactor. This is the end of the proof.

\section{Proof of Theorem 1.2}

To prove Theorem 1.2 we recall two auxiliary results. The first one was proved in [4] while the second one was proved in [15]. 
Lemma 4.1. Let $f$ be a polynomial and $f=\prod_{j=1}^{s} f_{j}^{\alpha_{j}}$ its decomposition into irreducible factors in $\mathbb{C}[x, y, z]$. Then $f$ is a Darboux polynomial if and only if all the $f_{j}$ are Darboux polynomials. Moreover, if $K$ and $K_{j}$ are the cofactors of $f$ and $f_{j}$, then $K=\sum_{j=1}^{s} \alpha_{j} K_{j}$.

Lemma 4.2. The existence of a rational first integral for a polynomial differential system (1.2) implies either the existence of a polynomial first integral, or the existence of two Darboux polynomials with the same non-zero cofactor.

Proof of Theorem 1.2. By Theorem 1.3, Lemma 4.1 and the non-existence of polynomial first integrals (see Corollary 1.1), it follows that every Darboux polynomial of system (1.2) is of the form $x^{m} y^{n} z^{l}$ with cofactor

$$
\begin{aligned}
& K=m\left(\rho\left(b_{1}+x\right)\left(b_{2}+y\right)-\frac{x}{k}\left(b_{1}+x\right)\left(b_{2}+y\right)-a_{1} y\left(b_{2}+y\right)\right)+ \\
& n\left(a_{1} x\left(b_{2}+y\right)-a_{2} z\left(b_{1}+x\right)-d_{1}\left(b_{1}+x\right)\left(b_{2}+y\right)\right)+ \\
& l\left(a_{2} y\left(b_{1}+x\right)-d_{2}\left(b_{1}+x\right)\left(b_{2}+y\right)\right),
\end{aligned}
$$

where $m, n, l$ are non-negative integers. From Lemma 4.2 and the non-existence of polynomial first integrals, the existence of a rational first integral implies the existence of two coprime Darboux polynomials with the same non-zero cofactor. So the first integral must be of the form $R / S=x^{m_{1}} y^{n_{1}} z^{l_{1}} /\left(x^{m_{2}} y^{n_{2}} z^{l_{2}}\right)$ with at least one of the integers $m_{1}-m_{2}, n_{1}-n_{2}$ and $l_{1}-l_{2}$, positive and at least one negative, and the cofactors of $R$ and $S$ must be equal.

According to equation (3.1), the equality of the cofactors of $R$ and $S$ imply that

$$
\begin{aligned}
& \left(m_{1}-m_{2}\right)\left(\rho\left(b_{1}+x\right)\left(b_{2}+y\right)-\frac{x}{k}\left(b_{1}+x\right)\left(b_{2}+y\right)-a_{1} y\left(b_{2}+y\right)\right)+ \\
& \left(n_{1}-n_{2}\right)\left(a_{1} x\left(b_{2}+y\right)-a_{2} z\left(b_{1}+x\right)-d_{1}\left(b_{1}+x\right)\left(b_{2}+y\right)\right)+ \\
& \left(l_{1}-l_{2}\right)\left(a_{2} y\left(b_{1}+x\right)-d_{2}\left(b_{1}+x\right)\left(b_{2}+y\right)\right)=0 .
\end{aligned}
$$

Computing the coefficient of $x^{3} y$ in (3.2) we deduce that $m_{1}=m_{2}$. Moreover, computing the coefficient of $x z$ in (3.2) we get that $n_{1}=n_{2}$. Therefore it follows from (3.2) that $l_{1}=l_{2}$. In short, there are no rational first integrals. This is the end of the proof.

\section{$5 \quad$ Preliminary results}

The equation defining the exponential factor $F=\exp (h / f)$ with cofactor $L$ as in (1.6) can be written as

$$
\dot{x} \frac{\partial}{\partial x}\left(\frac{h}{f}\right)+\dot{y} \frac{\partial}{\partial y}\left(\frac{h}{f}\right)+\dot{z} \frac{\partial}{\partial z}\left(\frac{h}{f}\right)=L,
$$


where we have simplified the common factor $F$, and $L$ is given in

$$
\begin{aligned}
L= & \beta_{0}+\beta_{1} x+\beta_{2} y+\beta_{3} z+\beta_{4} x^{2}+\beta_{5} x y+\beta_{6} x z+\beta_{7} y^{2}+\beta_{8} y z+\beta_{9} z^{2}+ \\
& \beta_{10} x^{3}+\beta_{11} x^{2} y+\beta_{12} x^{2} z+\beta_{13} x y^{2}+\beta_{14} x y z+\beta_{15} x z^{2}+\beta_{16} y^{3}+ \\
& \beta_{17} y^{2} z+\beta_{18} y z^{2}+\beta_{19} z^{3} .
\end{aligned}
$$

The following is a well-known result on exponential factors. Its proof is given in [3] or [4], see also [5].

Proposition 5.1. If $F=\exp (h / f)$ is an exponential factor for the polynomial differential system (1.2) and $f$ is not a constant polynomial, then $f=0$ is an invariant algebraic surface of system (1.2) with multiplicity higher than one.

When the exponential factor is of the form $\exp (h)$ then it is associated to the multiplicity of some invariant algebraic curve at infinity, see [5].

According to Theorem 1.3, Lemma 4.1 and Proposition 5.1, if system (1.2) has exponential factors, they must be of the form

$$
F=\exp \left(h /\left(x^{n_{1}} y^{n_{2}} z^{n_{3}}\right)\right),
$$

where $h \in \mathbb{C}[x, y, z]$ and $n_{1}, n_{2}, n_{3}$ are non-negative integers. We shall need the following auxiliary results.

\section{Exponential factors with $n_{1}=n_{2}=n_{3}=0$}

Lemma 6.1. The unique exponential factors (3.2) of system (1.2) with $n_{1}=n_{2}=$ $n_{3}=0$, are the ones described in Theorem 1.4.

Proof. Taking $F=\exp (h)$ and doing $y=0$ in (3.1) we have that

$$
b_{2}\left(b_{1}+x\right)\left[x\left(\rho-\frac{x}{k}\right) \frac{\partial \bar{h}}{\partial x}-d_{2} z \frac{\partial \bar{h}}{\partial z}\right]=\bar{L},
$$

where $\bar{h}$ is the restriction of $h$ to $y=0$, and $\bar{L}$ is the restriction of $L$ to $y=0$, i.e.

$$
\bar{L}=\beta_{0}+\beta_{1} x+\beta_{4} x^{2}+\beta_{10} x^{3}+z\left(\beta_{3}+\beta_{6} x+\beta_{9} z+\beta_{12} x^{2}+\beta_{15} x z+\beta_{19} z^{2}\right) .
$$

If we restrict (3.1) to $z=0$ and denote by $\hat{h}$ the restriction of $\bar{h}$ to $z=0$, we have that

$$
b_{2}\left(b_{1}+x\right) x\left(\rho-\frac{x}{k}\right) \frac{d \hat{h}}{d x}=\beta_{0}+\beta_{1} x+\beta_{4} x^{2}+\beta_{10} x^{3},
$$

which implies $\beta_{0}=0$ and

$$
\beta_{1} x+\beta_{4} x^{2}+\beta_{10} x^{3}=c_{0} x\left(b_{1}+x\right)(\rho-x / k), \quad c_{0} \in \mathbb{C},
$$

where $b_{2} d \hat{h} / d x=c_{0}$. Consequently

$$
\beta_{1}=b_{1} c_{0} \rho, \quad \beta_{4}=c_{0}\left(k \rho-b_{1}\right) / k, \quad \beta_{10}=-c_{0} / k .
$$


By (3.1) and (3.2) $b_{1}+x$ divides $\bar{L}$ and $\beta_{1} x+\beta_{4} x^{2}+\beta_{10} x^{3}$, since $\beta_{0}=0$ it also divides $\beta_{3}+\beta_{6} x+\beta_{9} z+\beta_{12} x^{2}+\beta_{15} x z+\beta_{19} z^{2}$, so

$$
\beta_{3}=b_{1}\left(\beta_{6}-b_{1} \beta_{12}\right), \quad \beta_{15}=\frac{\beta_{9}}{b_{1}}, \quad \beta_{19}=0 .
$$

On the other hand if we restrict (3.1) to $z=0$ and denote again by $\tilde{h}$ (respectively $\tilde{L}$ ) the restriction of $h$ (respectively $L$ ) to $z=0$, we get that

$$
\begin{array}{r}
\left(b_{2}+y\right)\left[x\left(\rho\left(b_{1}+x\right)-\frac{x}{k}\left(b_{1}+x\right)-a_{1} y\right) \frac{\partial \tilde{h}}{\partial x}+y\left(a_{1} x-d_{1}\left(b_{1}+x\right)\right) \frac{\partial \tilde{h}}{\partial y}\right]=\tilde{L}= \\
c_{0} x\left(b_{1}+x\right)(\rho-x / k)+y\left(\beta_{2}+\beta_{5} x+\beta_{7} y+\beta_{11} x^{2}+\beta_{13} x y+\beta_{16} y^{2}\right) .
\end{array}
$$

Clearly we have that $\tilde{L}$ must be divisible by $b_{2}+y$, then $c_{0}=0$ (i.e., $\beta_{1}=\beta_{4}=$ $\left.\beta_{10}=0\right)$ and

$$
\beta_{2}=b_{2}\left(\beta_{7}-b_{2} \beta_{16}\right), \quad \beta_{5}=b_{2} \beta_{13}, \quad \beta_{11}=0 .
$$

Hence we have that

$$
\begin{aligned}
L= & z\left(b_{1}+x\right)\left(b_{1} \beta_{6}-b_{1}^{2} \beta_{12}+b_{1} \beta_{12} x+\beta_{9} z\right)+y\left(b_{2}+y\right) \\
& \left(\beta_{7}-\beta_{16} b_{2}+\beta_{13} x+\beta_{16} y\right)+y z\left(\beta_{8}+\beta_{14} x+\beta_{17} y+\beta_{18} z\right) .
\end{aligned}
$$

Evaluating (3.1) on $x=-b_{1}$ and $y=-b_{2}$ (i.e. evaluating $L$ on $x=-b_{1}$ and $y=-b_{2}$ and equaling to zero), we get that

$$
b_{2}\left(\beta_{8}-b_{1} \beta_{14}+\beta_{17} b_{2}\right) z+\beta_{18} b_{2} z^{2}=0 .
$$

So

$$
\beta_{18}=0 \quad \text { and } \quad \beta_{8}=b_{1} \beta_{14}+b_{2} \beta_{17} .
$$

Thus $L$ has the form

$$
\begin{aligned}
L= & z\left(b_{1}+x\right)\left(b_{1} \beta_{6}-b_{1}^{2} \beta_{12}+b_{1} \beta_{12} x+\beta_{9} z\right)+y\left(b_{2}+y\right) \\
& \left(\beta_{7}-\beta_{16} b_{2}+\beta_{13} x+\beta_{16} y\right)+y z\left(b_{1} \beta_{14}+b_{2} \beta_{17}+\beta_{14} x+\beta_{17} y\right) .
\end{aligned}
$$

We write $h=\sum_{j=0}^{n} h_{j}(y, z) x^{j}$ where each $h_{j}$ is a polynomial in the variables $y$ and $z$. Computing the coefficient of $x^{n+2}$ in (3.1), if $n \geq 1$ we get

$$
-\frac{n}{k}\left(b_{2}+y\right) h_{n}(y, z)=0
$$

Therefore $h_{n}(y, z)=0$. Consequently $h=h_{0}(y, z)$.

We write $h_{0}=\sum_{j=0}^{n} h_{0, j}(y) z^{j}$ where each $h_{0, j}$ is a polynomial in the variable $y$.

If $n \geq 2$, then computing the coefficient of $z^{n+1}$ in (3.1) we have

$$
-a_{2} y\left(b_{1}+x\right) \frac{d h_{0, n}}{d y}=0, \quad \text { i.e. } \quad h_{0, n}=c_{0, n} \in \mathbb{C} .
$$


Now computing the coefficient of $z^{n}$ in equation (3.1), we obtain

$$
-a_{2} y\left(b_{1}+x\right) \frac{d h_{0, n-1}}{d y}+n\left(b_{1}+x\right)\left(a_{2} y-d_{2}\left(b_{2}+y\right)\right) c_{0, n}=0,
$$

if $n>2$. Since $d_{2} b_{2} \neq 0$, we get $c_{0, n}=0$, i.e., $h_{0, n}=0$ if $n>2$. So $n \leq 2$ and we write

$$
h_{0}=h_{0,0}(y)+h_{0,1}(y) z+h_{0,2}(y) z^{2} .
$$

Now substituting $h_{0}$ in (3.1) we get a polynomial of degree 3 in $z$ that we write

$$
\sum_{k=0}^{3} e_{k}(x, y) z^{k}=0
$$

Since $e_{3}(x, y)=-a_{2}\left(b_{1}+x\right) y h_{0,2}^{\prime}(y)=0$, it follows that $h_{0,2}(y)=m_{0} \in \mathbb{C}$. Then

$$
\begin{aligned}
e_{2}(x, y)= & -\frac{1}{b_{1}}\left(b_{1} \beta_{9}+2 b_{1}^{2} b_{2} d_{2} m_{0}+\left(\beta_{9}+2 b_{1} b_{2} d_{2} m_{0}\right) x-\right. \\
& \left.2 b_{1}^{2}\left(a_{2}-d_{2}\right) m_{0} y-2 b_{1}\left(a_{2}-d_{2}\right) m_{0} x y+a_{2} b_{1}\left(b_{1}+x\right) y h_{0,1}^{\prime}(y)\right)=0 .
\end{aligned}
$$

Solving this differential equation we get

$$
h_{0,1}(y)=\frac{1}{a_{2} b_{1}}\left(b_{1}\left(-2 d_{2} m_{0} y+a_{2}\left(2 m_{0} y+l_{0}\right)\right)-\left(\beta_{9}+2 b_{1} b_{2} d_{2} m_{0}\right) \log y\right) .
$$

Since $h_{0,1}(y)$ must be a polynomial, $\beta_{9}=-2 b_{1} b_{2} d_{2} m_{0}$. Then

$$
h_{0,1}(y)=l_{0}+\frac{2\left(a_{2}-d_{2}\right) m_{0}}{a_{2}} y .
$$

We have

$$
\begin{aligned}
e_{0}(x, y)= & b_{2}\left(\beta_{16} b_{2}-\beta_{7}\right) y-\beta_{13} b_{2} x y-\beta_{7} y^{2}-\beta_{13} x y^{2}-\beta_{16} y^{3}- \\
& y\left(b_{1} b_{2} d_{1}+a_{1} b_{2} x-b_{2} d_{1} x-b_{1} d_{1} y+a_{1} x y-d_{1} x y\right) h_{0,0}^{\prime}(y)=0 .
\end{aligned}
$$

Solving this differential equation and since we do not want independent terms in $h_{0,0}(y)$ (because they only change the exponential factor multiplying by a constant), we obtain

$$
h_{0,0}(y)=\frac{-2 \beta_{16} b_{2} y+2 \beta_{7} y+2 \beta_{13} x y+\beta_{16} y^{2}}{2\left(-b_{1} d_{1}+\left(a_{1}-d_{1}\right) x\right)} .
$$

We separate the proof in two cases.

Case 1: $d_{1} \neq a_{1}$. Since $h_{0,0}(y)$ must be a polynomial forcing that $h_{0,0}(y)=k_{1} y$ we get that

$$
\beta_{7}=-b_{1} d_{1} k_{1}, \quad \beta_{13}=a_{1} k_{1}-d_{1} k_{1}, \quad \beta_{16}=0 .
$$

Finally from $e_{1}(x, y)=0$, we have that

$$
k_{1}=-\frac{\beta_{14}+\left(d_{2}-a_{2}\right) l_{0}}{a_{2}}, \quad \beta_{17}=\beta_{12}=m_{0}=0, \quad \beta_{6}=-b_{2} d_{2} l_{0} .
$$


Hence

$$
h_{0}=-\frac{\beta_{14}+\left(d_{2}-a_{2}\right) l_{0}}{a_{2}} y+l_{0} z .
$$

So if $d_{1} \neq a_{1}$ the unique exponential factors (3.2) of system (1.2) with $n_{1}=n_{2}=$ $n_{3}=0$, are the ones described in Theorem 1.4(a).

Case 2: $d_{1}=a_{1}$. From (3.3) it follows that

$$
h_{0,0}(y)=-\frac{2\left(\beta_{7}-\beta_{16} b_{2}\right) y+2 \beta_{13} x y+\beta_{16} y^{2}}{2 b_{1} d_{1}} .
$$

We consider two subcases.

Subcase 2.1: $d_{2} \neq a_{2}$. Then solving $e_{1}(x, y)=0$ we get

$$
\begin{aligned}
& \beta_{6}=-b_{2} d_{2} l_{0}, \quad \beta_{12}=\beta_{13}=0, \quad \beta_{17}=\frac{a_{2} \beta_{16}}{a_{2}-d_{2}}, \quad m_{0}=-\frac{a_{2}^{2} \beta_{16}}{2 a_{1} b_{1}\left(a_{2}-d_{2}\right)^{2}}, \\
& \beta_{14}=\frac{a_{2} \beta_{7}}{a_{1} b_{1}}-\frac{a_{2} \beta_{16} b_{2}\left(a_{2}-2 d_{2}\right)}{a_{1} b_{1}\left(a_{2}-d_{2}\right)}-\left(d_{2}-a_{2}\right) l_{0}
\end{aligned}
$$

Therefore

$$
\begin{aligned}
h_{0}= & -\frac{\beta_{7}}{a_{1} b_{1}} y+l_{0} z+\frac{\beta_{16}}{2 a_{1} b_{1}\left(a_{2}-d_{2}\right)^{2}}\left(2 b_{2}\left(a_{2}-d_{2}\right)^{2} y-\right. \\
& \left.\left(a_{2}-d_{2}\right)^{2} y^{2}-2 a_{2}\left(a_{2}-d_{2}\right) y z-a_{2}^{2} z^{2}\right) .
\end{aligned}
$$

Hence if $d_{1}=d_{2}$ and $d_{2} \neq a_{2}$ the unique exponential factors (3.2) of system (1.2) with $n_{1}=n_{2}=n_{3}=0$, are the ones described in the first part of Theorem 1.4(b). Subcase 2.2: $d_{2}=a_{2}$. Now solving $e_{1}(x, y)=0$ we get

$$
\beta_{6}=-a_{2} b_{2} l_{0}, \quad \beta_{12}=\beta_{13}=\beta_{16}=\beta_{17}=0, \quad \beta_{14}=\frac{a_{2} \beta_{7}}{a_{1} b_{1}} .
$$

Then

$$
h_{0}=-\frac{\beta_{7}}{a_{1} b_{1}} y+l_{0} z+m_{0} z^{2} .
$$

Consequently if $d_{1}=d_{2}$ and $d_{2}=a_{2}$ the unique exponential factors (3.2) of system (1.2) with $n_{1}=n_{2}=n_{3}=0$, are the ones described in the second part of Theorem 1.4(b). This is the end of the proof.

\subsection{Darboux polynomials of system (1.2) restricted to $y=0$}

Equation (1.2) restricted to $y=0$ has the form

$$
\begin{aligned}
& \dot{x}=b_{2}\left(b_{1}+x\right) x\left(\rho-\frac{x}{k}\right) \\
& \dot{z}=-d_{2} b_{2}\left(b_{1}+x\right) z
\end{aligned}
$$

where the parameters $b_{1}, b_{2}, \rho, k$ are taken to be positive. 
Lemma 6.2. System (3.4) has no polynomial first integrals.

(a) If $\rho / d_{2}$ is irrational, then the unique irreducible Darboux polynomials with non-zero cofactor are $x, z, x+b_{1}$ and $x-k \rho$.

(b) If $\rho / d_{2}$ is rational, then system (3.4) has the rational first integral $H=$ $\frac{k \rho-x}{x} z^{-\rho / d_{2}}$, and consequently all its orbits are contained in invariant algebraic curves.

Proof. Note that system (3.4) after the rescaling of the time by the quantity $b_{2}\left(b_{1}+\right.$ $x)$ can be written as

$$
x^{\prime}=x\left(\rho-\frac{x}{k}\right), \quad z^{\prime}=-d_{2} z .
$$

Then any first integral must be a function of $H=\frac{k \rho-x}{x} z^{-\rho / d_{2}}$ and any function of the variable $H$ cannot be a polynomial. This concludes the proof of the lemma for the polynomial first integrals.

Clearly all the invariant curves of the system (3.5) are of the form $h x=(k \rho-$ $x) z^{-\rho / d_{2}}$ with $h \in \mathbb{R}$, and with the exception of $x=0$. Then statements (a) and (b) of the lemma follow easily. This is the end of the proof.

\subsection{Darboux polynomials of system (1.2) restricted to $z=0$}

Equation (1.2) restricted to $z=0$ has the form

$$
\begin{aligned}
& \dot{x}=x\left(b_{2}+y\right)\left(\rho\left(b_{1}+x\right)-\frac{x}{k}\left(b_{1}+x\right)-a_{1} y\right) \\
& \dot{y}=y\left(b_{2}+y\right)\left(a_{1} x-d_{1}\left(b_{1}+x\right)\right),
\end{aligned}
$$

where the parameters $a_{1}, b_{1}, b_{2}, d_{1}, k, \rho$ are positive.

Lemma 6.3. System (3.6) has no polynomial first integrals. Furthermore, the unique irreducible Darboux polynomials with non-zero cofactor are $x, y$ and $b_{2}+y$.

Proof. Let $f$ be a polynomial first integral of system (3.6) of degree $n \geq 1$. We can assume that $f$ has no constant terms. We consider two different cases.

Case 1. $f$ is not divisible by $y$. We write $f=\sum_{j=0}^{n} f_{j}(x) y^{j}$ where $f_{j}(x)$ is a polynomial in $x$ and $f_{0}(x) \neq 0$. Then $f_{0}$ satisfies (3.6) restricted to $y=0$ that is

$$
x b_{2}\left(b_{1}+x\right)\left(\rho-\frac{x}{k}\right) \frac{d f_{0}}{d x}=0 .
$$

Solving it we get $f_{0}=c_{0}$ and since $f$ has no constant terms, $f_{0}=0$, a contradiction.

Case 2. $f$ is divisible by $y$. We write $f=y^{l} g$ where $1 \leq l \leq n$ and $g$ is a polynomial which is not divisible by $y$. We write $g=\sum_{j=0}^{n-l} g_{j}(x) y^{j}$. Clearly $g_{0}(x) \neq 0$ 
and $g_{0}$ satisfies

$$
x b_{2}\left(b_{1}+x\right)\left(\rho-\frac{x}{k}\right) \frac{d g_{0}}{d x}=-l b_{2}\left(a_{1} x-d_{1}\left(b_{1}+x\right)\right) g_{0} .
$$

Solving it we get

$$
g_{0}=L x^{\frac{l d_{1}}{\rho}}\left(b_{1}+x\right)^{\frac{-a_{1} k}{b_{1}+k \rho}}(x-k \rho)^{-\frac{b_{1} d_{1}+\left(d_{1}-a_{1}\right) k \rho}{\rho\left(b_{1}+k \rho\right)}}, \quad L \in \mathbb{C} .
$$

Since $x+b_{1} \neq x-k \rho$ and $a_{1} k /\left(b_{1}+k \rho\right)>0$, we get that $g_{0}(x)$ is not a polynomial, a contradiction. This shows that system (3.6) has no polynomial first integrals.

Let $f$ be a Darboux polynomial of system (3.6). Then $f$ satisfies

$$
x\left(b_{2}+y\right)\left(\rho\left(b_{1}+x\right)-\frac{x}{k}\left(b_{1}+x\right)-a_{1} y\right) \frac{\partial f}{\partial x}+y\left(b_{2}+y\right)\left(a_{1} x-d_{1}\left(b_{1}+x\right)\right) \frac{\partial f}{\partial y}=K f,
$$

where

$$
K=\alpha_{0}+\alpha_{1} x+\alpha_{2} y+\alpha_{3} x^{2}+\alpha_{4} x y+\alpha_{5} y^{2}+\alpha_{6} x^{3}+\alpha_{7} x^{2} y+\alpha_{8} x y^{2}+\alpha_{9} y^{3} .
$$

We note that $x, z$ and $b_{1}+x$ are Darboux polynomials of system (3.6) with non-zero cofactor and of degree one. Furthermore any other invariant straight line (i.e. $f=0$ with $f=\gamma_{0}+\gamma_{1} x+\gamma_{2} y$ ) imply that some of the parameters in (3.6) must be zero, so they do not exist.

We assume that $f$ is an irreducible Darboux polynomial of system (3.7) with non-zero cofactor and with degree $n \geq 2$. From (3.7) and since $f$ is irreducible $b_{2}+y$ divides $K$ this implies that

$$
\alpha_{0}=\alpha_{2} b_{2}-\alpha_{5} b_{2}^{2}+\alpha_{9} b_{2}^{3}, \quad \alpha_{1}=\alpha_{4} b_{2}-\alpha_{8} b_{2}^{2}, \quad \alpha_{3}=\alpha_{7} b_{2}, \quad \alpha_{6}=0 .
$$

Consequently

$K=\left(b_{2}+y\right)\left(\alpha_{2}-\alpha_{5} b_{2}+\alpha_{9} b_{2}^{2}+\left(\alpha_{4}-\alpha_{8} b_{2}\right) x+\alpha_{7} x^{2}+\left(\alpha_{5}-\alpha_{9} b_{2}\right) y+\alpha_{8} x y+\alpha_{9} y^{2}\right)$.

On $x=0$ if we denote by $\bar{f}$ the restriction of $f$ to $x=0$, and restricting (3.7) to $x=0$, after rescaling by $b_{2}+y$, we get

$$
-y d_{1} b_{1} \frac{d \bar{f}}{d y}=\left(\alpha_{2}-\alpha_{5} b_{2}+\alpha_{9} b_{2}^{2}+\left(\alpha_{5}-\alpha_{9} b_{2}\right) y+\alpha_{9} y^{2}\right) \bar{f}
$$

Solving this differential equation we obtain

$$
\bar{f}=L \exp \left(-\frac{2\left(\alpha_{5}-\alpha_{9} b_{2}\right) y+\alpha_{9} y^{2}+2\left(\alpha_{2}-\alpha_{5} b_{2}+\alpha_{9} b_{2}^{2}\right) \log y}{2 b_{1} d_{1}}\right),
$$

with $L \in \mathbb{C}$. Since $\bar{f}$ must be a polynomial we have that $\alpha_{5}=\alpha_{9}=0$ and that $\alpha_{2}=-n_{1} d_{1} b_{1}$ for some positive integer $n_{1}$. Therefore

$$
K=-\left(b_{2}+y\right)\left(b_{1} d_{1} n_{1}+\left(\alpha_{8} b_{2}-\alpha_{4}\right) x-\alpha_{7} x^{2}-\alpha_{8} x y\right) .
$$


Now restricting (3.7) to $y=0$ and denoting by $\bar{h}$ the restriction of $h$ to $y=0$ we obtain, after simplifying by $b_{2}$, that

$$
x\left(\rho-\frac{x}{k}\right)\left(b_{1}+x\right) \frac{d \bar{h}}{d x}=-\left(b_{1} d_{1} n_{1}+\left(\alpha_{8} b_{2}-\alpha_{4}\right) x-\alpha_{7} x^{2}\right) \bar{h} .
$$

Then

$$
\begin{aligned}
\bar{h}= & M x^{-d_{1} n_{1} / \rho}\left(b_{1}+x\right)^{\left(k\left(\alpha_{4}-\alpha_{7} b_{1}-\alpha_{8} b_{2}+d_{1} n_{1}\right)\right) /\left(b_{1}+k \rho\right)} \\
& (-k \rho+x)^{\left(b_{1} d_{1} n_{1}-k \rho\left(\alpha_{4}-\alpha_{8} b_{2}+\alpha_{7} k \rho\right)\right) /\left(\rho\left(b_{1}+k \rho\right)\right)},
\end{aligned}
$$

where $M \in \mathbb{C}$. Since $\bar{h}$ must be a polynomial we get

$$
n_{1}=0, \quad \alpha_{4}=\alpha_{8} b_{2}+n_{2} \rho-\left(b_{1} n_{3}\right) / k \quad \text { and } \quad \alpha_{7}=-\left(n_{2}+n_{3}\right) / k,
$$

for some positive integers $n_{2}$ and $n_{3}$. The cofactor becomes

$$
K=\frac{1}{k} x\left(b_{2}+y\right)\left(-b_{1} n_{3}+k n_{2} \rho-\left(n_{2}+n_{3}\right) x+\alpha_{8} k y\right)
$$

and

$$
\bar{h}=M\left(b_{1}+x\right)^{n_{2}}(x-k \rho)^{n_{3}} .
$$

So

$$
f=M\left(b_{1}+x\right)^{n_{2}}(x-k \rho)^{n_{3}}+y g(x, y),
$$

for some polynomial $g(x, y)$.

Let $f_{n}=f_{n}(x, y)$ the homogeneous part of the polynomial $f$ of higher degree. Then, from (3.7) and (3.8), we have that

$$
x^{3} y \frac{d f_{n}}{d x}=\frac{1}{k}\left(x y\left(-n_{2} x-n_{3} x+\alpha_{8} k y\right)\right) f_{n} .
$$

Solving this differential equation we obtain

$$
f_{n}=N e^{-\left(\alpha_{8} y\right) / x} x^{-\left(n_{2}+n_{3}\right) / k}
$$

with $N \in \mathbb{C}$. Since $f_{n}$ is a homogeneous polynomial we must have $\alpha_{8}=0$. So $f_{n}=N x^{-\left(n_{2}+n_{3}\right) / k}$. Therefore $k=-\left(n_{2}+n_{3}\right) / n$ and $f_{n}=N x^{n}$. Now, from (3.9) it follows that $n=n_{2}+n_{3}$. Hence $k=-1$, a contradiction because $k$ is positive. In short there are no irreducible Darboux polynomial with non-zero cofactor of degree $n \geq 2$. This is the end of the proof.

\section{Proof of Theorems 1.4 and 1.5}

Proof of Theorem 1.4. Suppose that $F$ is an exponential factor. By (3.2) we have that $F$ is of the form $\exp \left(h / x^{n_{1}} y^{n_{2}} z^{n_{3}}\right)$ with $h \in \mathbb{C}[x, y, z]$ and $n_{1}, n_{2}, n_{3}$ are nonnegative integers and where $h$ is coprime with $x, y, z$. We assume that at least one of the $n_{1}, n_{2}$ or $n_{3}$ is positive and we will reach a contradiction. In view of (3.1) We have that $h$ satisfies

$$
\dot{x} \frac{\partial h}{\partial x}+\dot{y} \frac{\partial h}{\partial y}+\dot{z} \frac{\partial h}{\partial z}-\left(\frac{\dot{x}}{x} n_{1}+\frac{\dot{y}}{y} n_{2}+\frac{\dot{z}}{z} n_{3}\right) h=L x^{n_{1}} y^{n_{2}} z^{n_{3}}
$$


where we have simplified the common factor $\exp \left(h / x^{n_{1}} y^{n_{2}} z^{n_{3}}\right)$ and multiplied by $x^{n_{1}} y^{n_{2}} z^{n_{3}}$. We consider different cases.

Case 1: $n_{1}>0$. Taking $x=0$ in (3.1) and denoting by $\bar{h}$ the restriction of $h$ to $x=0$, we conclude that $\bar{h}$ satisfies

$$
\begin{aligned}
& -b_{1} y\left(d_{1}\left(b_{2}+y\right)+a_{2} z\right) \frac{\partial \bar{h}}{\partial y}+b_{1} z\left(a_{2} y-d_{2}\left(b_{2}+y\right)\right) \frac{\partial \bar{h}}{\partial z}= \\
& \left(n_{1}\left(b_{2}+y\right)\left(\rho b_{1}-a_{1} y\right)-n_{2} b_{1}\left(d_{1}\left(b_{2}+y\right)+a_{2} z\right)+n_{3} b_{1}\left(a_{2} y-d_{2}\left(b_{2}+y\right)\right)\right) \bar{h} .
\end{aligned}
$$

Since by hypothesis $h$ is coprime with $x$ we get that $\bar{h} \neq 0$. The part $-n_{1} a_{1} y^{2} \bar{h}$ of the left hand side of the previous equality has degree one more than the right hand side, so we have a contradiction.

Case 2: $n_{1}=0$ and $n_{2}>0$. Taking $y=0$ in (3.1) and denoting by $\hat{h}$ the restriction of $h$ to $y=0$, we conclude that $\hat{h}$ satisfies

$$
\begin{aligned}
& b_{2} x\left(b_{1}+x\right)(\rho-x / k) \frac{\partial \hat{h}}{\partial x}-b_{2} d_{2} z\left(b_{1}+x\right) \frac{\partial \hat{h}}{\partial z}= \\
& \left(n_{2}\left(a_{1} b_{2} x-\left(b_{1}+x\right)\left(b_{2} d_{1}+a_{2} z\right)\right)-n_{3} b_{2} d_{2}\left(b_{1}+x\right)\right) \hat{h} .
\end{aligned}
$$

Since by hypothesis $h$ is coprime with $y$ we get that $\hat{h} \neq 0$. Then equation (3.2) implies that $\hat{h}$ is a Darboux polynomial of system (1.2) restricted to $y=0$ with non-zero cofactor. From Lemma 6.2 we have two subcases.

Subcase 2.1: $\rho / d_{2} \notin \mathbb{Q}$. In this case $\hat{h}=R x^{m_{1}} z^{m_{2}}\left(b_{1}+x\right)^{m_{3}}(x-k \rho)^{m_{4}}$, with $R$ a constant and $m_{1}, m_{2}, m_{3}, m_{4}$ non-negative integers such that $m_{1}+m_{2}+m_{3}+m_{4}>$ 0 . Substituting $\hat{h}$ in (3.2) we get that

$$
\begin{aligned}
& m_{1} b_{2}\left(b_{1}+x\right)(\rho-x / k)-m_{2} b_{2} d_{2}\left(b_{1}+x\right)+m_{3} b_{2} x(\rho-x / k)-\frac{m_{4}}{k} b_{2} x\left(b_{1}+x\right) \\
& =n_{2}\left(a_{1} b_{2} x-\left(b_{1}+x\right)\left(b_{2} d_{1}+a_{2} z\right)\right)-n_{3} b_{2} d_{2}\left(b_{1}+x\right) .
\end{aligned}
$$

Computing the coefficient in (3.3) of the variable $x z$ we obtain $-a_{2} n_{2}=0$, a contradiction.

Subcase 2.2: $\rho / d_{2} \in \mathbb{Q}$. Let $\rho / d_{2}=u / v$ with $u, v \in \mathbb{N}$. By Lemma 6.2 all the Darboux polynomials of system (3.4) are of the form $\hat{h}=c^{v} x^{v} z^{u}-(k \rho-x)^{v}$ where $c$ is a constant. The cofactor of $\hat{h}$ is $-\frac{b_{2}}{k} v x\left(b_{1}+x\right)$. So, from (3.2),

$$
-\frac{b_{2}}{k} v x\left(b_{1}+x\right)=n_{2}\left(a_{1} b_{2} x-\left(b_{1}+x\right)\left(b_{2} d_{1}+a_{2} z\right)\right)-n_{3} b_{2} d_{2}\left(b_{1}+x\right) .
$$

So $n_{2} a_{2}=0$, a contradiction.

Case 3: $n_{1}=n_{2}=0$ and $n_{3}>0$. Taking $z=0$ in (3.1) and denoting by $\tilde{h}$ the restriction of $h$ to $z=0$, we conclude that $\tilde{h}$ satisfies

$$
\begin{aligned}
& x\left(b_{2}+y\right)\left(\rho\left(b_{1}+x\right)-\frac{x}{k}\left(b_{1}+x\right)-a_{1} y\right) \frac{\partial \tilde{h}}{\partial x}+y\left(b_{2}+y\right)\left(a_{1} x-d_{1}\left(b_{1}+x\right)\right) \frac{\partial \tilde{h}}{\partial y}= \\
& n_{3}\left(b_{1}+x\right)\left(a_{2} y-d_{2}\left(b_{2}+y\right)\right) \tilde{h} .
\end{aligned}
$$


Since by hypothesis $h$ is coprime with $z$ we get that $\tilde{h} \neq 0$. Then equation (3.4) implies that $\tilde{h}$ is a Darboux polynomial of system (1.2) restricted to $z=0$ with nonzero cofactor. By Lemma 6.3 we have $\tilde{h}=R x^{m_{1}}\left(b_{2}+y\right)^{m_{2}} y^{m_{3}}$, with $R$ a constant and $m_{1}, m_{2}, m_{3}$ non-negative integers such that $m_{1}+m_{2}+m_{3}>0$. Substituting $\tilde{h}$ in (3.4) we get that

$$
\begin{aligned}
& n_{3}\left(b_{1}+x\right)\left(a_{2} y-d_{2}\left(b_{2}+y\right)\right)=m_{1}\left(b_{2}+y\right)\left(\rho\left(b_{1}+x\right)-\frac{x}{k}\left(b_{1}+x\right)-a_{1} y\right)+ \\
& m_{2} y\left(a_{1} x-d_{1}\left(b_{1}+x\right)\right)+m_{3}\left(b_{2}+y\right)\left(a_{1} x-d_{1}\left(b_{1}+x\right)\right) .
\end{aligned}
$$

Computing the terms of degree $x^{2}$ in $(3.5)$ we get

$$
-\frac{m_{1}}{k}\left(b_{2}+y\right)=0 \quad \text { that is } \quad m_{1}=0 .
$$

Then, since $-d_{1} b_{1}+\left(a_{1}-d_{1}\right) x$ does not divide $b_{1}+x$ when $a_{1} \neq d_{1}$ we get from (3.5) that $n_{3}=0$, a contradiction. If $d_{1}=a_{1}$, then we have again a contradiction because the left hand side of the equality (3.5) depends on $x$ and the right hand side does not depend.

Case 4: $n_{1}=n_{2}=n_{3}=0$. We have $F=\exp (h)$ where $h \in \mathbb{C}[x, y, z]$. Then by Lemma 6.1 the theorem follows. This is the end of the proof.

The proof of the next lemma is given in [4].

Lemma 7.1. Suppose that system (1.2) defined in $\mathbb{R}^{n}$ of degree $m$ admits $p$ invariant algebraic surfaces $f_{i}=0$ with cofactors $K_{i}$ for $i=1, \ldots, p$ and $q$ exponential factors $F_{j}=\exp \left(g_{j} / h_{j}\right)$ with cofactors $L_{j}$ for $j=1, \ldots, q$. Then there exist $\lambda_{j}, \mu_{j} \in \mathbb{C}$ not all zero such that $\sum_{i=1}^{p} \lambda_{i} K_{i}+\sum_{i=1}^{q} \mu_{i} L_{i}=0$ if and only if the following real (multi-valued) function of Darboux type

$$
f_{1}^{\lambda_{1}} \cdots f_{p}^{\lambda_{p}} F_{1}^{\mu_{1}} \cdots F_{q}^{\mu_{q}}
$$

substituting $f_{i}^{\lambda_{i}}$ by $\left|f_{i}\right|^{\lambda_{i}}$ if $\lambda_{i} \in \mathbb{R}$, is a first integral of system (1.2)

Proof of Theorem 1.5. From Theorems 1.3, 1.4, Proposition 1.1 and Lemmas 4.1 and 7.1, if system (1.2) has a Darboux first integral $G$, then

$$
G=x^{\lambda_{1}} y^{\lambda_{2}} z^{\lambda_{3}} e^{\mu h} \quad \text { with } \quad \lambda_{1}, \lambda_{2}, \lambda_{3}, \mu \in \mathbb{C},
$$

with $h$ given in Theorem 1.4 and satisfying

$$
\lambda_{1} K_{1}+\lambda_{2} K_{2}+\lambda_{3} K_{3}+\mu L=0
$$

where

$$
\begin{aligned}
& K_{1}=\left(b_{2}+y\right)\left(\rho\left(b_{1}+x\right)-\frac{x}{k}\left(b_{1}+x\right)-a_{1} y\right), \\
& K_{2}=a_{1} x\left(b_{2}+y\right)-a_{2} z\left(b_{1}+x\right)-d_{1}\left(b_{1}+x\right)\left(b_{2}+y\right), \\
& K_{3}=\left(b_{1}+x\right)\left(a_{2} y-d_{2}\left(b_{2}+y\right)\right),
\end{aligned}
$$


and $L$ is given in Theorem 1.4. In view of Theorem 1.4 we consider different cases.

Case 1: $d_{1}=a_{1}$. In this case, using Theorem 1.4, (3.6) becomes

$$
\begin{aligned}
& \lambda_{1}\left(b_{2}+y\right)\left(\rho\left(b_{1}+x\right)-\frac{x}{k}\left(b_{1}+x\right)-a_{1} y\right)+\lambda_{2}\left(-a_{2} z\left(b_{1}+x\right)-a_{1} b_{1}\left(b_{2}+y\right)\right)- \\
& \lambda_{3} d_{2} b_{2}\left(b_{1}+x\right)+a y\left(-a_{2} z\left(b_{1}+x\right)-a_{1} b_{1}\left(b_{2}+y\right)\right)+ \\
& b z\left(b_{1}+x\right)\left(a_{2} y-d_{2}\left(b_{2}+y\right)\right)+c\left(-2 a_{1} b_{1} b_{2}^{2}\left(a_{2}-d_{2}\right)^{2} y+2 a_{1} b_{1}\left(a_{2}-d_{2}\right)^{2} y^{3}+\right. \\
& 2 a_{2} b_{1} b_{2}\left(a_{2}-d_{2}\right)\left(a_{1}-a_{2}+2 d_{2}\right) y z-2 a_{2} b_{2}\left(a_{2}-2 d_{2}\right)\left(a_{2}-d_{2}\right) x y z+ \\
& \left.2 a_{1} a_{2} b_{1}\left(a_{2}-d_{2}\right) y^{2} z+2 a_{2}^{2} b_{1} b_{2} d_{2} z^{2}+2 a_{2}^{2} b_{2} d_{2} x z^{2}\right)=0,
\end{aligned}
$$

where we have taken $\mu=1$ due to the arbitrariness of $a, b$ and $c$. The previous equality is a polynomial in $x, y, z$. Taking all the coefficients of the monomials of this polynomial equal to zero we get a system in the variables $\lambda_{1}, \lambda_{2}, \lambda_{3}, a, b, c$, $a_{1}, a_{2}, b_{1}, b_{2}, d_{1}, d_{2}, k, \rho$. It is easy to check that this system has no solutions except if either some of the coefficients $a_{1}, a_{2}, b_{1}, b_{2}, d_{1}, d_{2}, k, \rho$ are zero or $\lambda_{1}=\lambda_{2}=\lambda_{3}=a=b=c=0$. So in these cases there are no Darboux first integrals.

Case 2: $a_{1} \neq d_{1}$. In this case, using Theorem 1.4, (3.6) becomes case.

$$
\begin{aligned}
& \lambda_{1}\left(b_{2}+y\right)\left(\rho\left(b_{1}+x\right)-\frac{x}{k}\left(b_{1}+x\right)-a_{1} y\right)+ \\
& \lambda_{2}\left(a_{1} x\left(b_{2}+y\right)-a_{2} z\left(b_{1}+x\right)-d_{1}\left(b_{1}+x\right)\left(b_{2}+y\right)\right)+ \\
& \lambda_{3}\left(b_{1}+x\right)\left(a_{2} y-d_{2}\left(b_{2}+y\right)\right)+a y\left(a_{1} x\left(b_{2}+y\right)-a_{2} z\left(b_{1}+x\right)-d_{1}\left(b_{1}+x\right)\left(b_{2}+y\right)\right)+ \\
& b z\left(b_{1}+x\right)\left(a_{2} y-d_{2}\left(b_{2}+y\right)\right)=0,
\end{aligned}
$$

where we have taken $\mu=1$ due to the arbitrariness of $a$ and $b$. The previous equality is a polynomial in $x, y, z$. Taking all the coefficients of the monomials of this polynomial equal to zero we get a system in the variables $\lambda_{1}, \lambda_{2}, \lambda_{3}, a, b$, $a_{1}, a_{2}, b_{1}, b_{2}, d_{1}, d_{2}, k, \rho$. It is easy to check that this system has no solutions except if either some of the coefficients $a_{1}, a_{2}, b_{1}, b_{2}, d_{1}, d_{2}, k, \rho$ are zero or $\lambda_{1}=\lambda_{2}=\lambda_{3}=a=b=0$. So in these cases there are no Darboux first integrals. This is the end of the proof.

\section{References}

[1] M. Abramowitz and I.A. Stegun, Handbook of mathematical functions with formulas, graphs, and mathematical tables, National Bureau of Standards Applied Mathematics Series 55, U.S. Government Printing Office, Washington, D.C. 1964 .

[2] A. Bazykin, Nonlinear Dynamics of Interacting Populations, World Scientific, River Edge, NJ, 1998.

[3] C. Christopher and J. Llibre, Algebraic aspects of integrability for polynomial systems, Qualitative Theory of Dynamical Systems 1 (1999), 71-95. 
[4] C. Christopher and J. Llibre, Integrability via invariant algebraic curves for planar polynomial differential systems, Ann. Diff. Equations 16 (2000), 5-19.

[5] C. Christopher, J. Llibre and J.V. Pereira, Multiplicity of invariant algebraic curves in polynomial vector fields, Pacific J. Math. 229 (2007), 63-117.

[6] G. Darboux, Mémoire sur les équations différetielles algébraiques du premier ordre et du premier degreé (Métanges), Bull. Sci. Math. 2éme série 2 (1878), 60-96, 123-144, 151-200.

[7] G. Darboux, De l'emploi des solutions particulières algébriques dans l'intégration des systèmes d'équations différentielles algébriques, C. R. Math. Acad. Sci. Paris 86 (1878), 1012-1014.

[8] F. Dumortier, J. Llibre and J.C. Artés, Qualitative theory of planar differential systems, Universitext, Springer-Verlag, 2006.

[9] J. Écalle, Introduction deux fonctions analysables et preuve construtive de la conjecture de Dulac, Hermann, 1992.

[10] H. Freedman and J. So, Global stability and persistence of simple food chains, Math. Biosci. 76 (1985), 69-86.

[11] H. Freedman and P. Waltman, Mathematical analysis of some three-species food-chain models, Math. Biosci. 33 (1977), 257-276.

[12] T. Gard, Persistence in food chains with general interactions, Math. Biosci. 51 (1980), 165-174.

[13] A. Hastings and T. Powell, Chaos in a three species food chain, Ecol. 72 (1991), 896-903.

[14] Yu. A. Kuznetsov, O. De Feo and D. Rinaldi, Belyakov homoclinic bifurcations in a tritrophic food chain model, SIAM J. Appl. Math. 62 (2001), 462-487.

[15] J. Llibre and C. Valls, On the integrability of the Bianchi IX system, J. Math. Phys. 46 (2005), 072901-13pp

[16] J. Llibre and Xiang Zhang Darboux Theory of Integrability in $\mathbb{C}^{n}$ taking into account the multiplicity, J. of Differential Equations 246 (2009), 541-551.

[17] A. Lotka, Elements of Physical Biology, Williamsand Wilkins, Baltimore, MD, 1925.

[18] R. Moussu, Symétrie et forme normale des centes et foyers dégénérés, Ergodic Theory and Dynamical Systems 2 (1982), 241-251.

[19] J.D. Murray, Mathematical biology I. An introduction, third edition, Interdiciplinary Applied Mathematics 17, Springer-Verlag, New York, 2002. 
[20] S. Muratori and S. Rinaldi, Low and high frequency oscillations in threedimensional food chain systems, SIAM J. Appl. Math. 52 (1992), 1688-1706.

[21] V.V. Nemytskii and V.V Stepanov, Qualitative theory of differential equations, Dover Publ., New York, 1989.

[22] A. Seidenberg, Reduction of singularities of the differential equations $A d y=B d x$, Amer. J. Math. 90 (1968), 248-269.

[23] V. Volterra, Variazioni e fluttuazioni del numer di individui in specie animali conviventi, Mem. Acad. Lincei 2 (1926), 31-113. 